\title{
Turbulence-Chemistry Interaction in Lean Premixed Hydrogen Combustion
}

\author{
A. J. Aspden ${ }^{1,2}$, M. S. Day ${ }^{2}$ and J. B. Bell ${ }^{2}$ \\ ${ }^{1}$ Centre for Fluid Mechanics and Scientific Computing, Cranfield University, Bedfordshire, MK43 OAL, UK \\ ${ }^{2}$ Center for Computational Sciences and Engineering, Lawrence Berkeley National Laboratory, Berkeley, CA 94720, USA
}

\begin{tabular}{|c|c|c|}
\hline \multirow{2}{*}{$\begin{array}{r}\text { corresponding author: } \\
\text { address: }\end{array}$} & \multicolumn{2}{|l|}{ A. J. Aspden } \\
\hline & \multicolumn{2}{|c|}{$\begin{array}{l}\text { Centre for Fluid Mechanics and Scientific Computing } \\
\text { School of Engineering (B83) }\end{array}$} \\
\hline email: & \multicolumn{2}{|c|}{ a.j.aspden@cranfield.ac.uk } \\
\hline colloquium: & \multicolumn{2}{|c|}{ Turbulent flames } \\
\hline keywords: & \multicolumn{2}{|c|}{$\begin{array}{l}\text { turbulent combustion, premixed hydrogen } \\
\text { flames, low Mach number combustion }\end{array}$} \\
\hline \multicolumn{3}{|c|}{ Word count using method 2} \\
\hline & Table 1 & 58 \\
\hline & Table 2 & 203 \\
\hline & Figure 1 & 416 \\
\hline & Figure 2 & 210 \\
\hline & Figure 3 & 401 \\
\hline & Figure 4 & 485 \\
\hline & Figure 5 & 443 \\
\hline & Figure 6 & 273 \\
\hline & Figure 7 & 161 \\
\hline & - & - \\
\hline \multicolumn{2}{|c|}{ Tables and figures } & 2650 \\
\hline \multicolumn{2}{|c|}{ Combined text } & 3460 \\
\hline & - & - \\
\hline & Total & 6200) \\
\hline
\end{tabular}




\begin{abstract}
This paper presents three-dimensional direct numerical simulations of lean premixed hydrogen flames at an equivalence ratio of $\varphi=0.4$ over a range of turbulence levels from Ka $=1$ to 36 . The simulations form part of a larger effort to construct a DNS database that can be used by the community for model construction and validation. We have focussed on producing well-resolved simulations at conditions representative of atmospheric laboratory-scale flames. After an overview of phenomenological trends with increasing Karlovitz number, we examine the factors that lead to an observed decorrelation between fuel consumption and heat release in the flame at $\mathrm{Ka}=36$. We show that in this flame the fuel consumption is greatly enhanced in regions of positive curvature. We also show that the radical pool is enriched throughout the entire flame as Ka is increased. In particular, we identify three reactions that, driven by high molar concentrations of radicals at low temperatures, are responsible for high levels of heat release away from regions of fuel consumption, thereby accounting for the observed decorrelation between fuel consumption and heat release.
\end{abstract}




\section{Introduction}

There has been considerable recent interest in hydrogen or hydrogen-rich mixtures obtained from gasification of coal or biomass. These types of fuels provide clean alternatives to traditional petroleum and natural gas fuels. Burning under lean conditions reduces the exhaust gas temperatures, and consequently, thermal $\mathrm{NO}_{\mathrm{x}}$ emissions.

At low-to-moderate levels of turbulence, the thermodiffusive instability leads to flames that burn in wellknown cellular structures, which results in localised hot spots where the flame is burning more intensely due to local enrichment of fuel by diffusion [1-6]. At high turbulence levels, hydrogen flames transition to the distributed burning regime where the thermodiffusive instability is dominated by turbulent mixing [7, 8].

In the present paper, we present simulations of lean premixed hydrogen over a range of low-to-moderate turbulence levels. The simulations form part of a larger effort to construct a DNS database that can be used by the community for model construction and validation. We have focussed on producing well-resolved simulations with conditions that are representative of atmospheric laboratory-scale flames, rather than the more highly turbulent conditions of distributed flames.

We consider lean premixed hydrogen flames at an equivalence ratio $\varphi=0.4$. The characterisation of the turbulence in terms of the flame structure is given by the Karlovitz and Damköhler numbers. In Aspden et al. [9], we note that a freely-propagating lean hydrogen flame is distinctly different from the idealised flat laminar flame because of the thermodiffusive instability. We showed that a flame speed and thickness defined from a freely-propagating flame provide a better normalisation of flame behaviour than the laminar values. Thus, following [9] we work with freely-propagating Karlovitz and Damköhler numbers, defined as

$$
\mathrm{Ka}^{2}=\frac{\check{u}^{3}}{s_{F}^{3}} \frac{l_{F}}{l} \quad \text { and } \quad \mathrm{Da}=\frac{s_{F}}{\check{u}} \frac{l}{l_{F}}
$$

respectively, where $\check{u}$ and $l$ are the turbulent rms velocity and integral length scale, respectively, and $s_{F}$ and $l_{F}$ are the freely-propagating flame speed and width, respectively. 
For the present simulations, we consider an idealised configuration with a fixed domain width and integral length scale. Turbulence in the fluid is driven via a time-dependent zero-mean volumetric fluid forcing term $[8,10]$. The resulting turbulence intensity is controlled by adjusting the amplitude of the forcing.

We briefly sketch the computational methodology and describe the present study in section 2 . In section 3 we present the results of the simulations, starting with an overview of the observations and trends with Karlovitz number in section 3.1, and the identification of an interesting decorrelation between fuel consumption and heat release, which is explored further in section 3.2. We then examine the individual reactions that contribute to this decorrelation in section 3.3, and conclude with an interpretation of the results in section 4 .

\section{Computational Methodology}

The simulations presented here are based on a low Mach number formulation of the reacting flow equations. The methodology treats the fluid as a mixture of perfect gases. We use a mixture-averaged model for differential species diffusion and ignore Soret, Dufour, gravity and radiative transport processes. With these assumptions, the low Mach number equations for an open domain are

$$
\begin{aligned}
& \frac{\partial(\rho \boldsymbol{u})}{\partial t}+\nabla \cdot(\rho \boldsymbol{u u})=-\nabla \pi+\nabla \cdot \tau+\rho \boldsymbol{F}, \\
& \frac{\partial\left(\rho Y_{i}\right)}{\partial t}+\nabla \cdot\left(\rho Y_{i} \boldsymbol{u}\right)=\nabla \cdot\left(\rho \mathcal{D}_{i} \nabla Y_{i}\right)-\dot{\omega}_{i}, \\
& \frac{\partial(\rho h)}{\partial t}+\nabla \cdot(\rho h \boldsymbol{u})=\nabla \cdot\left(\frac{\lambda}{c_{p}} \nabla h\right)+ \\
& \sum_{i} \nabla \cdot\left[h_{i}\left(\rho \mathcal{D}_{i}-\frac{\lambda}{c_{p}}\right) \nabla Y_{i}\right],
\end{aligned}
$$

where $\rho$ is the density, $\boldsymbol{u}$ is the velocity, $\pi$ is the perturbational pressure, $Y_{i}$ is the mass fraction of species $i$, $h$ is the mass-weighted enthalpy of the gas mixture, $T$ is the temperature, and $\dot{\omega}_{i}$ is the net destruction rate for species $i$ due to chemical reactions. Also, $\lambda$ is the thermal conductivity, $\tau$ is the stress tensor, $c_{p}$ is the specific heat of the mixture, and $h_{i}(T)$ and $\mathcal{D}_{i}$ are the enthalpy and mixture-averaged diffusion coefficients of species $i$, respectively. Here $\boldsymbol{F}$ is a long-wavelength forcing term designed to establish and maintain 
turbulence with the desired properties. These evolution equations are supplemented by an equation of state for a perfect gas mixture. The reader is referred to [11] for details of the low Mach number model and its numerical implementation. The chemical kinetics and transport are modelled using the Li et al. hydrogen mechanism [12], which consists of 9 species with 19 fundamental reactions.

The overall numerical scheme converges with second-order accuracy in both space and time. The performance of the scheme for direct numerical simulation of premixed flame systems in regimes comparable to the present study was examined in [10]. An effective Kolmogorov length scale was formulated, which measures the actual Kolmogorov length scale realised in a simulation at a given resolution. Here, the most computationally demanding simulation, having the highest turbulence levels, has a computational cell width that is approximately 0.75 times the Kolmogorov length scale. At this resolution, the numerical scheme produces an effective Kolmogorov length scale that is just 3\% larger than the analytical value; the other simulations are better resolved. Also, the grid spacing for all cases considered is such that 25 cells span the thermal thickness of a freely-propagating flame corresponding to this fuel-air mixture; note that there are over 40 cells across the unstrained flat laminar flame. Thus, the flame and the turbulence are numerically well-resolved in all four of the cases we considered here.

\subsection{Simulation Configuration}

We simulated four downward-propagating flames in a high aspect ratio domain (8:1), with periodic lateral boundary conditions, a free-slip base and outflow at the top. The momentum source term that maintains the background turbulence results in a time-dependent zero-mean velocity field. It was shown in [10] that this approach gives approximately 10 integral length scales across the domain width. The equivalence ratio in all four cases was set to $\varphi=0.4$. For this mixture, the freely-propagating flame speed $s_{F} \approx 47 \mathrm{~cm} / \mathrm{s}$ and thermal thickness $l_{F} \approx 410$ microns, [9]. By contrast, a flat unstrained flame at this mixture propagates with speed $s_{L} \approx 22 \mathrm{~cm} / \mathrm{s}$ and thermal thickness $l_{L} \approx 680$ microns. The domain width, $L$, was chosen to ensure 
that approximately 25 computational cells span $l_{F}$ and 1024 cells span the domain. Thus, $L=1.64 \mathrm{~cm}$, giving an integral length scale, $l \approx 1.64 \mathrm{~mm}$. For these conditions, the integral scale is then four times $l_{F}$ for all four cases. An inert calculation was run to establish the turbulence at reduced expense, and the reacting flow simulation was initialised by superimposing a laminar flame solution onto the turbulent velocity field. The base grid in each of the four cases was $256 \times 256 \times 2048$. Two levels of grid refinement were added once the flame had become established, resulting in an effective resolution of $1024 \times 1024 \times 8096$. Details of the four cases are summarised in table 1.

\section{Results}

We first present an overview of the general behaviour and observations of the flames and trends with Karlovitz number. In particular, we note a decorrelation between the fuel consumption rate and the heat release. We explore the conditions leading to this decorrelation, and follow with a closer examination of a number of specific chemical reactions that we can attribute as the root cause.

\subsection{Overview}

In figure 1, we present representative slices of temperature, fuel consumption rate and heat release for the four cases (note that the range for the respective fields is the same for each Karlovitz number). In the low Ka case, the thermodiffusive instability leads to the usual cellular burning pattern, with stronger burning in regions of positive curvature (i.e. centre of curvature in the products), and large regions of fluid that are above the adiabatic flame temperature (dark red). As expected, we see an increase in flame complexity with increasing Ka; individual structures are smaller and appear to have a thinner flame (especially fuel consumption), but the total volume of reacting fluid is much greater. The peak fuel consumption rate and heat release increase with $\mathrm{Ka}$, and the local extinction events (breaks in the flame surface) observed at low $\mathrm{Ka}$ are less pronounced at high $\mathrm{Ka}$; turbulence mitigates the tendency for the thermodiffusive instability to 
extinguish the flame in regions of negative curvature.

\subsection{Decorrelation of fuel consumption and heat release}

A striking observation from figure 1 is that there appears to be a decorrelation of fuel consumption and heat release as the Karlovitz number increases. At $\mathrm{Ka}=1$ and 4 , the fuel consumption and heat release are reasonably well-correlated (there is a clear similarity between the two fields), but for $\mathrm{Ka}=36$, the regions with high positive curvature present relatively low levels of heat release, despite high levels of fuel consumption. An example of this is highlighted in the detail panels of a $0.26 \mathrm{~cm}$ square shown in magenta. Note that adjacent to the regions of high positive curvature, there are regions of relatively intense heat release with low levels of fuel consumption. These observations suggest a potential disruption of the flame chemistry.

The decorrelation is confirmed in the joint probability density function (JPDF) for fuel consumption rate and heat release, shown in figure 2 for $\mathrm{Ka}=1$ and 36 . The peak fuel consumption rate has approximately doubled (note the increased range on the abscissa), but the peak heat release has increased by a factor of four (as seen on the ordinate), and this intense heat release coincides with relatively low levels of fuel consumption.

To examine this observation in more detail, separate JPDFs of fuel consumption rate and heat release rate are plotted in figures $3(\mathrm{a}, \mathrm{d})$ and $3(\mathrm{~b}, \mathrm{e})$, respectively, where temperature has been used as a loose measure of progress through the flame. Note there is a small amount of high intensity heat release occurring at low temperature $(T<900 \mathrm{~K})$ in the $\mathrm{Ka}=36$ case, but there does not appear to be a corresponding response in the fuel consumption rate.

By taking the first moment with respect to heat release (the first moment with respect to $x_{1}$ is defined as $x_{1} P\left(x_{1}, x_{2}\right)$, where $P\left(x_{1}, x_{2}\right)$ is the JPDF of $x_{1}$ and $\left.x_{2}\right)$, the amount of heat released can be compared directly, figure 3(c,f); these plots are on the same scale so that the same colour corresponds to the same 
amount of heat release. It is clear that there is significantly more heat being released at the higher Karlovitz number, and that the heat release extends to much lower temperatures than in the low Karlovitz case, although the intense heat release observed at low temperatures does not contribute significantly to the overall heat release.

\subsection{Chemistry}

To investigate which reactions are responsible for the decorrelation between fuel consumption rate and heat release, we first present, in figure 4 , slices of the local heat release rate for six reactions that contribute the most to the overall heat release. For reference, table 2 presents the 19 fundamental reactions from the Li et al. mechanism [12]. We note that all slices in figure 4 are plotted on the same scale and that there is non-trivial positive heat release from reactions 7, 10, 12, 14 and 16, which are not shown. At $\mathrm{Ka}=1$, all of the reactions appear to be correlated; the peak reaction rate occurs in regions of positive curvature, and all reactions can be seen to be greatly reduced in the regions between the cellular burning structures. At $\mathrm{Ka}=36$, it is interesting to note that, locally, the enhancement of heat release is a small factor (approximately 2), but it is the enhanced volume of burning due to turbulence that is responsible for the enhancement to the overall heat release. Naturally, it can be seen that the heat release from reaction 3 is visually similar to the fuel consumption rate in figure 1; this is the main reaction for the breakdown of molecular hydrogen. By contrast, reactions 9, 11 and 13 show intense heat release in regions of negative curvature, and would appear to correlate visually with the heat release from figure 1; these reactions do not involve molecular hydrogen and so do not contribute directly to the fuel consumption.

To examine the heat release rate in more detail, we consider the contribution by individual reactions to the total rate of heat release as a function of temperature, shown in figure 5(a). To normalise the effect of the enhanced volume of burning at $\mathrm{Ka}=36$, the curves have been scaled by the peak heat release rate from reaction 3 at the respective Karlovitz number. This normalisation represents rate of heat release via each 
reaction for each mole of fuel that is consumed (up to a multiplicative factor). Note that the peak heat release from reaction 3 for $\mathrm{Ka}=36$ is approximately 5.7 times greater than for $\mathrm{Ka}=1$, i.e. without normalising in this manner, the curves for $\mathrm{Ka}=36$ would be 5.7 times bigger than $\mathrm{Ka}=1$. It is interesting to note that reactions 1 and 3, the main reactions responsible for the breakdown of molecular oxygen and hydrogen, respectively, are similar at both Karlovitz numbers. Reaction 8 appears to be relatively enhanced at high temperatures (around $1400 \mathrm{~K}$ ). Reaction 9, in particular, appears to be enhanced at low temperatures (below $900 \mathrm{~K}$ ), which also seems to be the case for reactions 11 and 13 , but to a lesser extent.

For a more direct comparison, the difference in heat release rates between the high and low Karlovitz cases is plotted in figure 5(b). This diagnostic provides a measure of the relative enhancement of heat release due to individual reactions at $\mathrm{Ka}=36$ compared with $\mathrm{Ka}=1$. The enhancement of the heat release rate due to reaction 8 around the adiabatic flame temperature is more pronounced here, and there is a clear enhancement of reactions $3,8,9$, and 13 also around the same temperature. The relative enhancement of heat release at low temperatures due to reactions 9,11 and 13 is also more pronounced here. There also appears to be a relative reduction in heat release at temperatures slightly above and below the adiabatic flame temperature, which is what would be expected from higher heat release in a narrow temperature range around the adiabatic flame temperature.

We compute the total heat release rate by summing together the rates due to all reactions. The enhancement of total heat released is plotted in figure 5(c). The overall effect of the changes in reactions is to increase the heat release rate at lower temperatures (due primarily to reactions 9, 11 and 13), and to increase the rate near the adiabatic flame temperature (due primarily to reactions $8,3,9$ and 13). We also observe a decreased relative heat release rate at temperatures slightly above and below the adiabatic flame temperature. Assuming that the negative-positive-negative profile observed around the adiabatic flame temperature is due to a narrower band of more intense heat release at $\mathrm{Ka}=36$, we can consider the relative change in heat release rates at low and high temperatures defined by the crossover point at $992 \mathrm{~K}$. Integrating the change in 
heat release above and below this crossover results in an relative enhancement of heat release in both cases, but the enhancement is approximately seven times greater at low temperature than at high temperature.

To explore the reason for this observed chemical response, JPDFs of molar concentration and temperature are presented in figure 6 for four of the radicals at $\mathrm{Ka}=1$ and $\mathrm{Ka}=36$; note that the first moment has been taken with respect to molar concentration so that the colour denotes the actual molar concentration rather than just the probability. For all radicals, the higher turbulence case has higher peak molar concentrations. However, for radicals $\mathrm{H}$ and $\mathrm{O}$ in particular, there is significantly higher volume of radicals at temperatures in excess of the adiabatic flame temperature at the lower turbulence level; the low Ka flame forms cellular structures (larger than in the high Ka case), in which temperatures are higher and have greater molar concentrations of radicals. Importantly, at low temperatures there is an enhanced radical pool in the high Ka case; the distributions are both broader and greater in magnitude than the low Ka case.

The enhanced radical pool is quantified more clearly in figure 7, where the ratio of mean molar concentration is plotted as a function temperature; this has been calculated by finding the mean molar concentration as a function of temperature $\bar{C}_{\mathrm{Ka}}(T)$ and evaluating the ratio $\bar{C}_{36} / \bar{C}_{1}$. Taking the mean of the molar concentration at each temperature accounts for the enhanced volume of burning in the high Ka case; however, without more careful conditioning, the relatively large volume of pure fuel and pure product dominates this ratio at the inflow and adiabatic flame temperatures. Hence we present the enhancement ratio only over the range $350 \mathrm{~K}$ to $1350 \mathrm{~K}$. It is clear that the mean molar concentration of all five radicals is greater in the high Ka case across the entire temperature range, but especially for $\mathrm{H}$, which is about three times higher, and for $\mathrm{O}$ and $\mathrm{OH}$, which are about twice as high.

\section{Discussion and Conclusions}

We have presented high-resolution direct numerical simulations of lean premixed hydrogen flames at an equivalence ratio of $\varphi=0.4$ over a range of turbulence levels ranging from $\mathrm{Ka}=1$ to $\mathrm{Ka}=36$. At low 
Ka, large cellular structures expected from low Lewis number flames were observed, along with temperatures far exceeding the adiabatic flame temperature, interspersed by local (near) extinction events. As the Karlovitz number was increased, these large structures became much less apparent, with a broad distribution of smaller-scale structures that burned more intensely, with a thinner flame, and the local extinction events were much less apparent.

A striking observation was a decorrelation between fuel consumption and heat release at high Ka; fuel was consumed primarily in regions of high positive curvature but the heat release occurred in regions of negative curvature adjacent to the regions of fuel consumption.

An analysis of heat released by individual reactions confirmed that the high Ka flame burned more intensely than the low Ka flame. Furthermore, low-probability high-intensity burning was identified at low temperatures in the high Ka case, which was attributed to enhancement of reactions 9,11 and 13 at temperatures below about $800 \mathrm{~K}$. While the observed local changes are clear in the diagnostics, the global effect on the flame remains quite small; for example, at $\mathrm{Ka}=1$, the heat release that takes place below $800 \mathrm{~K}$ is approximately $11 \%$ of the total, and increases to $15 \%$ at $\mathrm{Ka}=36$.

While an examination of the detailed transport mechanisms responsible for the observed turbulencechemistry interaction is beyond the scope of the present paper, we present a speculative interpretation of the process. Turbulence folds the flame into smaller structures, where the relative high mobility of molecular hydrogen leads to enhanced fuel consumption in regions of positive curvature, and greatly enhances the radical pool at temperatures around the adiabatic flame temperature. These radicals are then transported, either by molecular diffusion or by turbulent mixing, both to lower and to slightly higher temperatures. Above the adiabatic flame temperature, an enhancement in reaction 8 was observed, leading to greater heat release. Since turbulence inhibits the formation of large-scale celluar structures, this enhanced heat release around the adiabatic flame temperature is restricted to a narrower temperature range than at low Ka. The increased availability of radicals at low temperatures, especially $\mathrm{H}$, but also $\mathrm{O}, \mathrm{OH}$, and $\mathrm{HO}_{2}$, was found to 
increase reactions 9,11 and 13 , which all produced sufficiently significant increase in heat release in regions away from positive flame curvature to result in a decorrelation of fuel consumption and overall heat release.

Since the effects observed here are likely to be driven (at least in part) by molecular diffusion, the omission of Soret and Dufour effects in the model will be relevant. A recent study [13] underscores the role of these terms on the quantitative flame propagation characteristics in a freely-propagating (no turbulence) two-dimensional setting. However, it was also shown that the simpler mixture-averaged transport formulation retained much of the qualitative behaviour of these flames, and there is no reason to suggest that the same is not true in the present setting. Moving to a full multicomponent transport model is a current and interesting area of future investigation, although we note that increased computational cost may render such a study infeasible in the present context.

\section{Acknowledgements}

This work was supported by the DOE Applied Mathematics Research Program of the DOE Office of Advanced Scientific Computing Research under the U.S. Department of Energy Contract No. DE-AC02$05 \mathrm{CH} 11231$.

\section{References}

[1] Y. B. Zeldovich, Theory of Combustion and Detonation in Gases (in Russian), Acad. Sci. USSR, 1944.

[2] G. H. Markstein, The Journal of Chemical Physics 17 (4) (1949) 428-429.

[3] B. Bregeon, A. S. Gordon, F. A. Williams, Combustion and Flame 33 (1978) 33-45.

[4] T. Mitani, F. A. Williams, Combust. Flame 39 (1980) 169-190.

[5] M. Baum, T. J. Poinsot, D. C. Haworth, N. Darabiha, J. Fluid Mech. 281 (1994) 1-32.

[6] M. S. Day, J. B. Bell, P.-T. Bremer, V. Pascucci, V. Beckner, M. J. Lijewski, Combustion and Flame 156 (5) (2009) 1035 1045.

[7] M. J. Dunn, A. R. Masri, R. B. Bilger, Combustion and Flame 151 (1-2) (2007) 46 - 60.

[8] A. Aspden, M. Day, J. Bell, JFM 680 (2011) 287-320.

[9] A. Aspden, M. Day, J. Bell, Proceedings of the Combustion Institute 33 (1) (2011) 1463 - 1471.

[10] A. J. Aspden, N. Nikiforakis, S. B. Dalziel, J. B. Bell, Comm. App. Math. Comput. Sci. 3 (1) (2008b) 101.

[11] M. S. Day, J. B. Bell, Combust. Theory Modelling 4 (2000) 535-556.

[12] J. Li, Z. Zhao, A. Kazakov, F. L. Dryer, International Journal of Chemical Kinetics 36 (10) (2004) 566-575.

[13] J. F. Grcar, J. B. Bell, M. S. Day, Proceedings of the Combustion Institute 32 (1) (2009) 1173-1180. 


\begin{tabular}{l|c|c|c|c} 
Case & A & B & C & D \\
\hline$\check{u}[\mathrm{~m} / \mathrm{s}]$ & 0.75 & 1.9 & 3.9 & 8.2 \\
$\check{u} / s_{F}$ & 1.6 & 4.0 & 8.3 & 17 \\
$\mathrm{Ka}_{F}$ & 1.0 & 4.0 & 12 & 36 \\
$\mathrm{Da}_{F}$ & 2.5 & 1.0 & 0.48 & 0.23
\end{tabular}

Table 1: Turbulence properties for the four simulations. 


\begin{tabular}{|c|c|}
\hline \# & Reaction \\
\hline 1 & $\mathrm{H}+\mathrm{O}_{2}=\mathrm{O}+\mathrm{OH}$ \\
\hline 2 & $\mathrm{O}+\mathrm{H}_{2}=\mathrm{H}+\mathrm{OH}$ \\
\hline 3 & $\mathrm{H}_{2}+\mathrm{OH}=\mathrm{H}_{2} \mathrm{O}+\mathrm{H}$ \\
\hline 4 & $\mathrm{O}+\mathrm{H}_{2} \mathrm{O}=\mathrm{OH}+\mathrm{OH}$ \\
\hline 5 & $\mathrm{H}_{2}+\mathrm{M}=\mathrm{H}+\mathrm{H}+\mathrm{M}$ \\
\hline 6 & $\mathrm{O}+\mathrm{O}+\mathrm{M}=\mathrm{O}_{2}+\mathrm{M}$ \\
\hline 7 & $\mathrm{O}+\mathrm{H}+\mathrm{M}=\mathrm{OH}+\mathrm{M}$ \\
\hline 8 & $\mathrm{H}+\mathrm{OH}+\mathrm{M}=\mathrm{H}_{2} \mathrm{O}+\mathrm{M}$ \\
\hline 9 & $\mathrm{H}+\mathrm{O}_{2}(+\mathrm{M})=\mathrm{HO}_{2}(+\mathrm{M})$ \\
\hline 10 & $\mathrm{HO}_{2}+\mathrm{H}=\mathrm{H}_{2}+\mathrm{O}_{2}$ \\
\hline 11 & $\mathrm{HO}_{2}+\mathrm{H}=\mathrm{OH}+\mathrm{OH}$ \\
\hline 12 & $\mathrm{HO}_{2}+\mathrm{O}=\mathrm{O}_{2}+\mathrm{OH}$ \\
\hline 13 & $\mathrm{HO}_{2}+\mathrm{OH}=\mathrm{H}_{2} \mathrm{O}+\mathrm{O}_{2}$ \\
\hline 14 & $\mathrm{HO}_{2}+\mathrm{HO}_{2}=\mathrm{H}_{2} \mathrm{O}_{2}+\mathrm{O}_{2}$ \\
\hline 15 & $\mathrm{HO}_{2}+\mathrm{HO}_{2}=\mathrm{H}_{2} \mathrm{O}_{2}+\mathrm{O}_{2}$ \\
\hline 16 & $\mathrm{H}_{2} \mathrm{O}_{2}(+\mathrm{M})=\mathrm{OH}+\mathrm{OH}+(\mathrm{M})$ \\
\hline 17 & $\mathrm{H}_{2} \mathrm{O}_{2}+\mathrm{H}=\mathrm{H}_{2} \mathrm{O}+\mathrm{OH}$ \\
\hline 18 & $\mathrm{H}_{2} \mathrm{O}_{2}+\mathrm{H}=\mathrm{HO}_{2}+\mathrm{H}_{2}$ \\
\hline 19 & $\mathrm{H}_{2} \mathrm{O}_{2}+\mathrm{O}=\mathrm{OH}+\mathrm{HO}_{2}$ \\
\hline 20 & $\mathrm{H}_{2} \mathrm{O}_{2}+\mathrm{OH}=\mathrm{HO}_{2}+\mathrm{H}_{2} \mathrm{O}$ \\
\hline 21 & $\mathrm{H}_{2} \mathrm{O}_{2}+\mathrm{OH}=\mathrm{HO}_{2}+\mathrm{H}_{2} \mathrm{O}$ \\
\hline
\end{tabular}

Table 2: The reactions of the Li et al. mechanism [12]. 


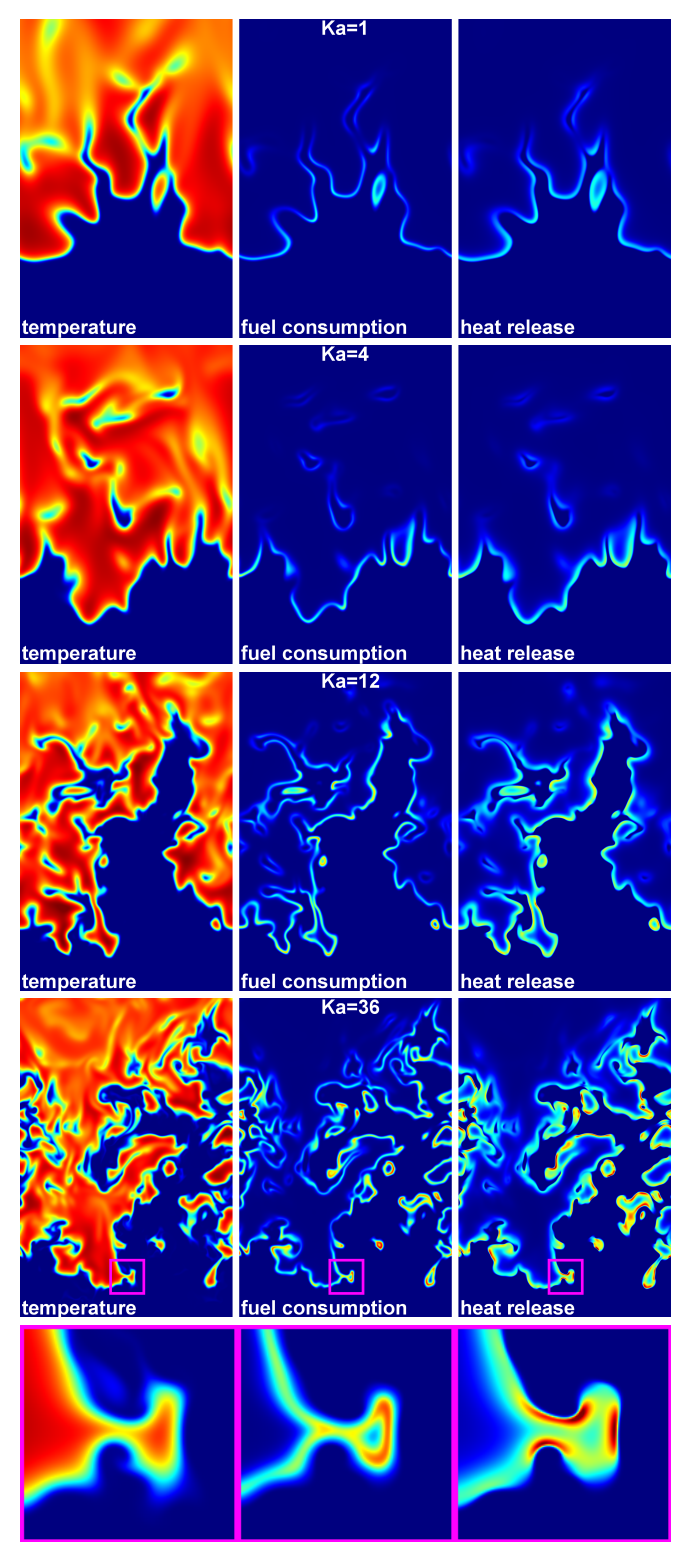

Figure 1: Representative slices of temperature, fuel consumption rate and heat release for all four simulations. As the turbulence is increased, the large-scale cellular burning structures become disrupted, leading to a broader distribution of small-scale structures, which burn more intensely. Note how, at high Ka, there is a decorrelation between fuel consumption and heat release that is not observed at low Ka. The decorrelation is highlighted in the detail panels (bottom row), which correspond to the $0.26 \mathrm{~cm}$ square highlighted in magenta. 

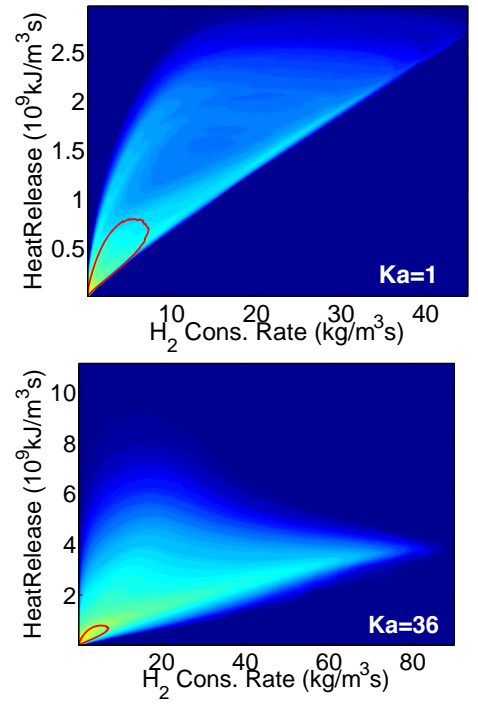

Figure 2: JPDFs of heat release and fuel consumption for $\mathrm{Ka}=1$ and 36 . The red line denotes the mean path through the freely-propagating flame, plotted as a parametric function of temperature. There is a clear enhancement of fuel consumption and heat release with increasing Ka. In both, we observe regions where high levels of heat release correspond to low levels of fuel consumption. 

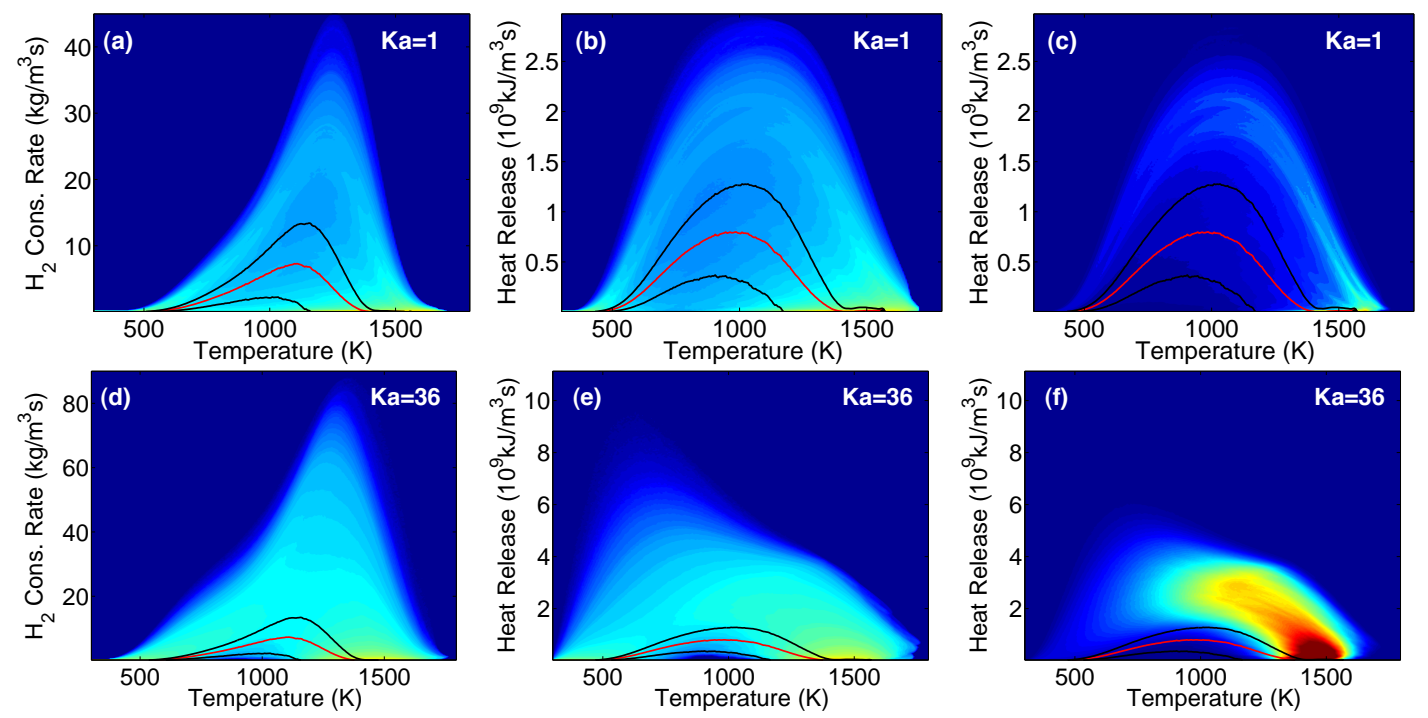

Figure 3: (a,d) JPDF of fuel consumption and temperature. Note the general similarity in distribution, but a doubling in peak amplitude. (b,e) JPDF of heat release and temperature. Note the greatly enhanced heat release at low temperatures for high Ka. (c,f) JPDF of heat release and temperature where the first moment has been taken with respect to heat release; these are plotted on the same scale such that the colour corresponds to comparable of heat release rates. (a-c) $\mathrm{Ka}=1$. (d-f) $\mathrm{Ka}=36$. In all cases, the red line denotes the mean of the freely-propagating flame, and the black lines denote one standard deviation around the mean. 


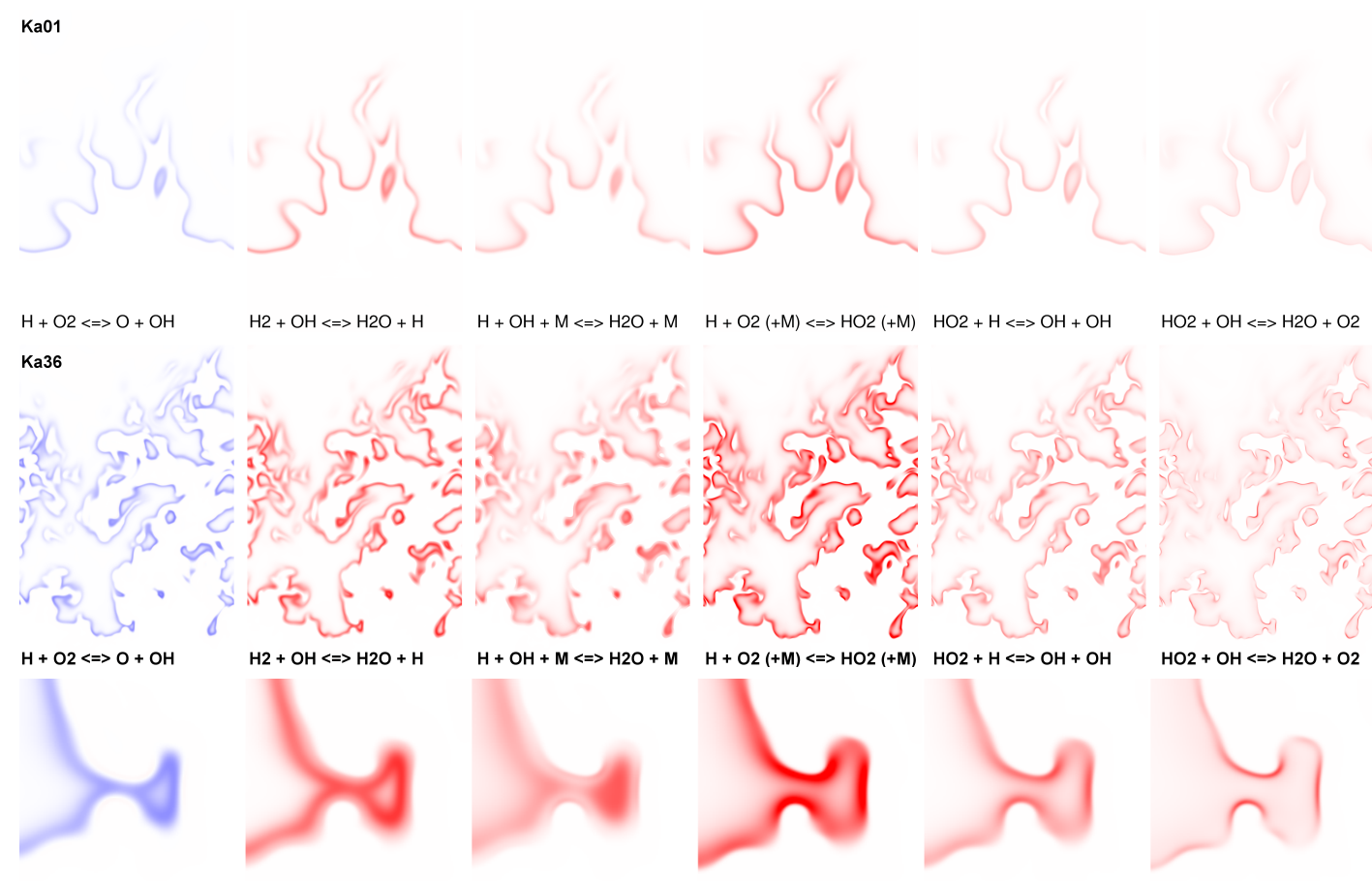

Figure 4: Slices of individual heats of reactions for the most energetic reactions (left-to-right: reactions 1, 3, 8, 9, 11 and 13) at $\mathrm{Ka}=1$ (top row) and $\mathrm{Ka}=36$ (middle row) with detail from the highlighted region in figure 1 for $\mathrm{Ka}=36$ (bottom row), exothermic and endothermic reactions in red and blue, respectively. The natural correlation between reaction 3 and the fuel consumption rate (figure 1) is evident. At high Ka, note the correlation between the heat release from reactions 9,11 and 13 with the total heat release in figure 1. 

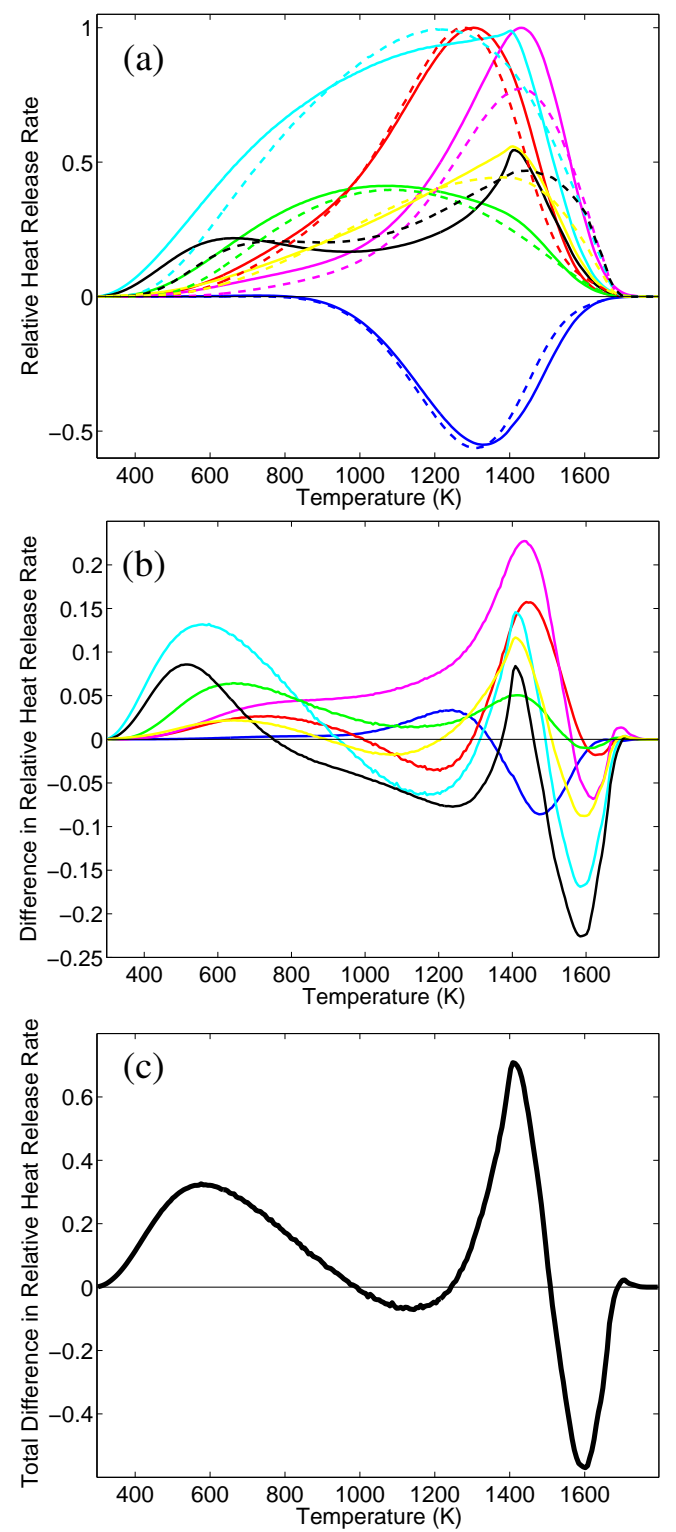

Figure 5: (a) Heat release rate by individual reaction versus temperature, normalised by the peak heat release rate of reaction 3 for the respective Ka. Solid and dashed lines represent $\mathrm{Ka}=36$ and 1, respectively. The colours represent different reactions: (blue:R01) $\mathrm{H}+\mathrm{O}_{2}=\mathrm{O}+\mathrm{OH}$; (red:R03) $\mathrm{H}_{2}+\mathrm{OH}=\mathrm{H}_{2} \mathrm{O}+\mathrm{H}$; (magenta:R08) $\mathrm{H}+\mathrm{OH}+\mathrm{M}=\mathrm{H}_{2} \mathrm{O}+\mathrm{M}$; (cyan:R09) $\mathrm{H}+\mathrm{O}_{2}(+\mathrm{M})=\mathrm{HO}_{2}(+\mathrm{M})$; (green:R11) $\mathrm{HO}_{2}+\mathrm{H}=\mathrm{OH}+\mathrm{OH}$; (black:R13) $\mathrm{HO}_{2}+\mathrm{OH}=\mathrm{H}_{2} \mathrm{O}+\mathrm{O}_{2}$; (yellow) sum of the remaining reactions. (b) The difference in relative heat release from each reaction between the high and low turbulence cases. Colours as in (a). Note that reactions 9,11 and 13 are enhanced at low temperatures. (c) Total difference in relative heat release as a function of temperature. 

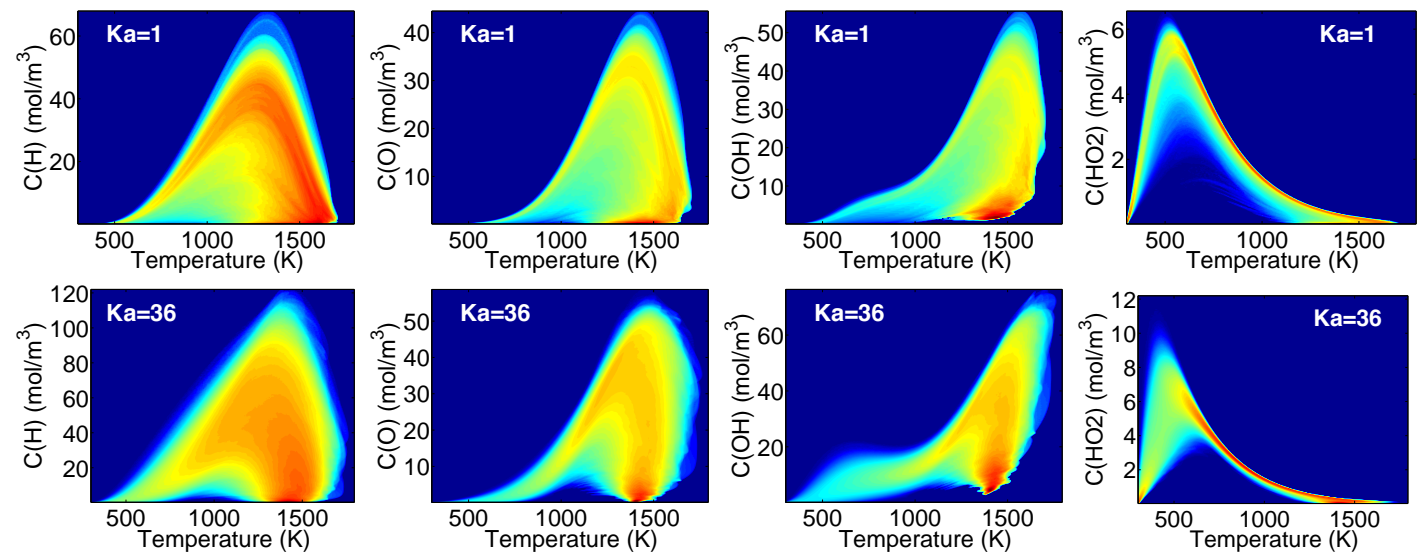

Figure 6: JPDF of molar concentration and temperature where the first moment has been taken with respect to molar concentration. Note in particular the higher molar concentration at lower temperatures in the high Ka case. 


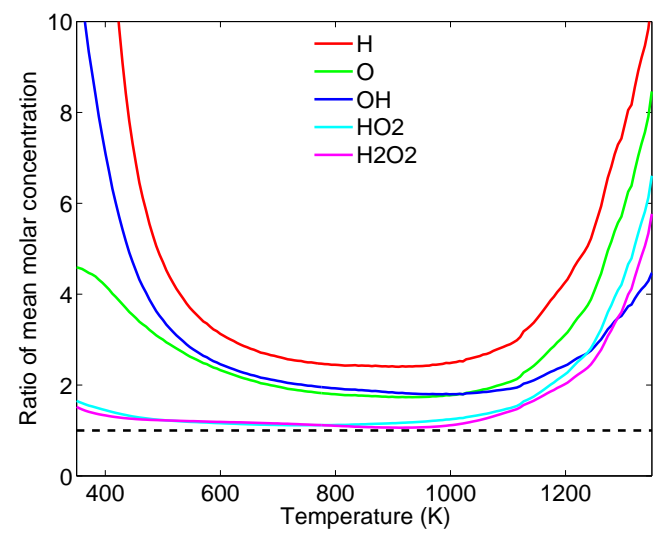

Figure 7: Ratio of mean molar concentration $\bar{C}_{36} / \bar{C}_{1}$, plotted against temperature. The range is $350 \mathrm{~K}$ to $1350 \mathrm{~K}$ as the volume of pure fuel and pure product distorts the mean. Note there is an enhancement of the radical pool across the entire temperature range plotted, with a factor of 3 observed for $\mathrm{H}$, and 2 for $\mathrm{O}$ and $\mathrm{OH}$. 